\title{
COMPLETIONS OF CERTAIN NILPOTENT GROUPS
}

\author{
SIEGFRIED MORAN and JANET WILLIAMS
}

(Received 14 June 1978; revised 1 May 1979)

Communicated by H. Lausch

\begin{abstract}
The completions of certain nilpotent groups with respect to some ascending sequences of integral domains are constructed. These completions are generalizations of Lazard completions for the groups under consideration and they are Lie algebras over the first integral domain in the sequence. The construction is possible in particular for finite $p$-groups of exponent $p$ and class $<p$.
\end{abstract}

1980 Mathematics subject classification (Amer. Math. Soc.): 20 E I5.

Given a finitely generated group $G$ of a certain type we show how to construct a completion $G\left(\Omega_{i}\right)$ of it with respect to a sequence of appropriate integral domains

$$
\boldsymbol{\Omega}_{1} \subseteq \boldsymbol{\Omega}_{2} \subseteq \ldots \subseteq \boldsymbol{\Omega}_{i} \subseteq \ldots
$$

This works in particular for a finite $p$-group of exponent $p$ and class $<p$. We start off by considering the case of torsion-free groups. Here the construction is known when every $\Omega_{i}=\Omega_{1}$ is a binomial ring. It is due to $P$. Hall (1957), $\S 6$, and our method makes fundamental use of his construction. (Developments of some parts of Hall's paper can also be found in Duguid '(1957) and Warfield (1976).) Besides Hall (1957), §6, we assume that the reader is familiar with Lazard (1954). For the groups here considered, their Lazard completions are completions with a particular choice of the sequence $\left(\Omega_{i}\right)$. It follows from results of Lazard (1954) that $G\left(\Omega_{i}\right)$ is a Lie algebra over $\Omega_{1}$. Finally, we note that we also make use of a method given in Moran (1973) for constructing a set of free generators for certain subalgebras of free Lie algebras.

We would like to record our gratitude to Brian Hartley for a number of useful remarks which helped us overcome some local difficulties. 


\section{Completions of torsion-free nilpotent groups}

Let $G$ be a torsion-free finitely generated nilpotent group. Suppose that

$$
G=G_{1} \supset G_{2} \supset \ldots \supset G_{j} \supset \ldots
$$

is a central series in $G$ such that every $G_{j} / G_{j+1}$ is torsion-free. Now for every $j$ one can choose a finite set of elements of $G_{j}$ so that modulo $G_{j+1}$ they form a basis for the abelian group $G_{j} / G_{j+1}$. We take the union of all such bases to give $u_{1}, u_{2}, \ldots, u_{n}$. In this ordering we write an element of the basis for $G_{j}$ modulo $G_{j+1}$ before an element of the basis for $G_{j+1}$ modulo $G_{j+2}$. Then every element of $G$ has a unique representation of the form

$$
u_{1}^{\alpha_{1}} u_{2}^{\alpha_{2}} \ldots u_{n}^{\alpha_{n}} \text {, }
$$

where $\alpha_{1}, \alpha_{2}, \ldots, \alpha_{n}$ are integers. We call the above set of elements $u_{1}, u_{2}, \ldots, u_{n}$ a canonical basis for $G$.

Let $x$ and $y$ be elements of $G$. Then

$$
x=u_{1}^{\alpha_{1}} u_{2}^{\alpha_{2}} \ldots u_{n}^{\alpha_{n}} \quad \text { and } \quad y=u_{1}^{\beta_{1}} u_{2}^{\beta_{2}} \ldots u_{n}^{\beta_{n}} .
$$

For every integer $\lambda$ we have that

and

$$
x^{\lambda}=u_{1}^{\gamma_{1}} u_{2}^{\gamma_{2}} \ldots u_{n}^{\gamma_{n}}
$$

$$
x y=u_{1}^{\delta_{1}} u_{2}^{\delta_{2}} \ldots u_{n}^{\delta_{n}}
$$

We have a function $\mu$ defined as follows. $\mu(i)$ is the positive integer such that $u_{i}$ belongs to $G_{\mu(i)}$ but $u_{i}$ does not belong to $G_{\mu(i)+1}$, for all $i$. By $Q_{j}$ we denote the ring of rational numbers $r / s$ such that $r$ and $s$ are coprime and the prime divisors of $s$ do not exceed $j$.

The following result gives information about the functions $\gamma_{i}$ and $\delta_{i}$. It is a refinement of Hall (1957), Theorem 6.5. We omit the proof as it is similar to the one given by Hall. One only needs to introduce the obvious refinements in the induction hypotheses.

THEOREM 1. The functions $\gamma_{i}$ and $\delta_{i}$ are integer-valued polynomials in the arguments $\alpha_{1}, \alpha_{2}, \ldots, \alpha_{n}, \lambda$ and $\alpha_{1}, \alpha_{2}, \ldots, \alpha_{n}, \beta_{1}, \beta_{2}, \ldots, \beta_{n}$ respectively. The constant terms of the polynomials are zero and the coefficients belong to $Q_{\mu(i)}$. This holds for $i=1,2, \ldots, n$.

We are now in a position to give completions of torsion-free finitely generated nilpotent groups which are generalizations of the construction of Hall (1957). Let

$$
\mathbf{\Omega}_{1} \subseteq \mathbf{\Omega}_{\mathbf{2}} \subseteq \ldots \subseteq \mathbf{\Omega}_{c}
$$


where $c$ is the class of $G$, be an ascending chain of integral domains such that

$$
Q_{j} \subseteq \Omega_{j} \text { for all } j .
$$

By $G\left(\Omega_{i}\right)$ we denote the set of all elements of the form $\prod_{i=1}^{n} u_{i}^{\alpha_{i}}$, where $u_{1}, u_{2}, \ldots, u_{n}$ is a canonical basis for the torsion-free finitely generated nilpotent group $G$ and $\alpha_{i}$ belongs to $\Omega_{\mu(i)}$ for all $i$. Then as in Hall (1957), §6, one can prove

THEOREM 2. If the product of two elements of $G\left(\Omega_{i}\right)$ is defined by the polynomials $\delta_{i}$ of Theorem 1 , then $G\left(\Omega_{i}\right)$ is a group. If exponentiation by an element of $\Omega_{1}$ is defined by the polynomials $\gamma_{i}$, then $G\left(\Omega_{i}\right)$ is an $\Omega_{1}$-powered group.

One only has to note that the above assertions reduce to polynomial identities which are true for all integer values of the arguments. The definition of a powered group can be found in Hall (1957), §6.

\section{Subgroups of free nilpotent groups generated by original commutators}

We begin by defining original commutators and original Lie monomials.

Definition 1. Let $F$ denote a free nilpotent group of class $c$. By ${ }^{i} F$ we denote the $i$ th member of the lower central series of $F$. A set of elements of $F$ which are linearly independent modulo ${ }^{2} F$ is called a set of original commutators (in $F$ ) of weight 1. Suppose that original commutators (in $F)$ of weight $<i(<c)$ have been chosen. Let $B(i)$ denote the smallest isolated subgroup of $F$ which contains ${ }^{i+1} F$ and all complex commutators of the following type. It has more than one component, the arguments vary over all original commutators of weight less than $i$ and the sum of these weights (in each case) is $i$. A set of elements of ${ }^{i} F$ which is linearly independent modulo $B(i)$ is called a set of original commutators (in $F$ ) of weight $i$.

There is a similar concept for a free group $\mathscr{F}$ - original commutators (in $\mathscr{F}$ ) of weight $i(\leqslant c)$.

We consider the free group $\mathscr{F}$ to be naturally embedded in the Magnus algebra $\mathscr{M}$ over the field $Q$ of rational numbers. If a set of original commutators in $\mathscr{F}$ is given, then the non-constant least degree monomial terms of these original commutators are called original Lie monomials. Their weights are defined to be the weights of the corresponding original commutators.

There are obvious ways of going from original commutators in $\mathscr{F}$ to original commutators in $\mathscr{F} /^{c+1} \mathscr{F}$ and back again.

LEMMA 1. A set of original Lie monomials freely generate a free Lie ring in the Magnus algebra $\mathscr{M}$. 
Proor. Take the generators of $\mathscr{M}$ and form the free Lie algebra $\mathscr{L}$ over $Q$ generated by them in $\mathscr{M}$. The subspace of $\mathscr{L}$ which consists of all homogeneous Lie elements of degree $i$ will be denoted by $\mathscr{L}_{(i)}$. Let $S$ denote the subalgebra of $\mathscr{L}$ generated by all the chosen original Lie monomials. Clearly $S \cap \mathscr{L}_{(1)}$ is spanned by the original Lie monomials of weight 1 . They are linearly independent. For otherwise, an ordered nontrivial power product of the original commutators (in $\mathscr{F})$ of weight 1 belongs to ${ }^{2} \mathscr{F}$, which contradicts the definition of original commutators of weight 1 .

Form all the Lie monomials on "the original Lie monomials of weight less than $i$ ". We choose those Lie monomials which belong to $\mathscr{L}_{(i)}$. They will span a vector subspace $S(i)$ in $\mathscr{L}_{(i)}$. Then $S \cap \mathscr{L}_{(i)}$ has a basis consisting of the original Lie monomials of weight $i$ modulo $S(i)$, since

$$
Q \otimes\left(\left.{ }^{i} \mathscr{F}\right|^{i+1} \mathscr{F}\right) /\left(\left.B(i)\right|^{i+1} \mathscr{F}\right) \cong \mathscr{L}_{(i)} / S(i)
$$

Hence, by Moran (1973), Theorem 2.1, we have that $S$ is a free Lie algebra with the original Lie monomials as a set of free generators. However, every original Lie monomial belongs to the free Lie $L$ ring generated in $\mathscr{M}$ by the generators of the Magnus algebra $\mathscr{K}$. Hence the original Lie monomials freely generate a free Lie subring of $L$.

We now give a definition of weighted basic commutators and weighted basic Lie monomials.

DEFINITION 2. A weighted basic commutator in $F$ is a basic commutator on some set of original commutators in $F$. Its absolute weight, which we denote by w( ), is the sum of the weights of the component original commutators. We will only consider weighted basic commutators of absolute weight not exceeding $c$. Weighted basic commutators are of course ordered according to their usual weight, that is, the number of components.

We also have a similar concept in a free group. Weighted basic Lie monomials and their absolute weights are defined analogously.

We are now in a position to establish the following fundamental

LEMMA 2. Let $\left\{b_{v}, v \in N\right\}$ be the set of weighted basic commutators corresponding to a given finite set of original commutators in a free nilpotent group $F$ of class $c$. Then

(i) every set $H_{j}$ of all ordered products $\dagger$

$$
\prod_{v} b_{v}^{m_{v}}
$$

† The ordering in an ordered product is by weight. 
where all $m_{v}$ are integers and $m_{\mathfrak{\tau}}=0$ if $\mathbf{w}\left(b_{\mathfrak{\tau}}\right)<j$, is a subgroup of

$$
\left\langle b_{v}, v \in N\right\rangle=H \text {; }
$$

(ii) $H_{1}=H$;

(iii) $H_{j}=H \cap^{\prime} F$ for all $j$;

(iv) $\left\{H_{j}\right\}$ is a Lazard $N$-series in $H$;

(v) every element of $H$ has a unique representation in the form given by (i) above;

(vi) every $H_{j} / H_{j+1}$ is a free abelian group.

Proof. Properties (i) and (ii) follow from the usual commutator collecting process (see, for instance, Hall (1959), Chapter 11).

(iii) Clearly $H_{j} \subseteq H \cap{ }^{j} F$ for all $j$. Suppose that $b$ belongs to $H \cap{ }^{j} F$ with $b$ belonging to $H_{i}$ but not belonging to $H_{i+1}$ for some $i \leqslant j$. Suppose that contrary to the assertion of the lemma we have that $i \neq j$. Let $\mathscr{F}$ denote the free group such that $\mathscr{F} / l^{c+1} \mathscr{F}=F$. Let $\bar{b}$ denote an inverse image of $b$ under the natural homomorphism $\mathscr{F} \rightarrow F$. Then

$$
\bar{b}+\mathscr{H}_{i+1}=1+\mathscr{M}_{i+1},
$$

since ${ }^{k} \mathscr{F} \subseteq 1+\mathscr{M}_{k}$ (see Lazard (1954), Théorème (4.2)), where $\mathscr{M}_{k}$ is the ideal of the Magnus algebra consisting of all those elements whose homogeneous components of degree $<k$ are zero. Now $b=\prod \dot{b}_{v}^{m_{v}}$ with $m_{v}=0$ when $\mathbf{w}\left(b_{v}\right)<i$. Hence

$$
\sum m_{\tau} \tilde{b}_{\tau}=0,
$$

where $\left\{\tilde{b}_{\mathfrak{t}}, \tau \in N(i)\right\}$ denotes the set of all weighted basic Lie monomials of absolute weight $i$ and not all integers $m_{\tau}$ are equal to zero. This contradicts Lemma 1.

(iv) This follows at once from (iii).

(v) Suppose that contrary to the assertion of the lemma we have that

$$
b=\prod_{v} b_{v}^{m_{v}}=e
$$

and not all $m_{v}$ equal zero. Let $b_{\tau}$ be a weighted basic commutator of lowest absolute weight such that $m_{\tau} \neq 0$. Suppose that $\mathbf{w}\left(b_{\tau}\right)=i$. Now one can proceed as in the proof of (iii) to arrive at a contradiction to Lemma 1.

(vi) Suppose that $b$ belongs to $H_{j}$ but does not belong to $H_{j+1}$. Further suppose that

$$
\left(b \cdot H_{j+1}\right)^{n}=H_{j+1}
$$

for some integer $n$. Then

$$
b=\prod_{\tau} b_{\tau}^{m_{\tau}} \text { modulo } H_{j+1} \text {, }
$$


where $w\left(b_{\mathfrak{r}}\right)=j$ for all $\tau$ and some integer $m_{\mathfrak{\imath}} \neq 0$. So

$$
e=b^{n}=\prod_{\tau} b_{\tau}^{n m_{\tau}} \cdot \prod_{v} b_{v}^{m_{v}}
$$

where every $w\left(b_{v}\right) \geqslant j+1$. Hence using the commutator collecting process in order to get the weighted basic commutators occurring in the above product in the right order, one gets that

$$
e=\ldots b_{t}^{n m_{\mathbf{v}}} \ldots
$$

for all $b_{\tau}$ with $\mathrm{w}\left(b_{\tau}\right)=j$. So the above proved property (v) shows that $n=0$, since some $m_{\tau} \neq 0$.

We now form the $\left(\Omega_{i}\right)$-completion of the above given group $H$ and of its subgroups $H_{j}$ in the way described in Theorem 2 and the explanation above it. The following result holds because it can be reduced, using Theorem 1, to certain polynomial identities, which are true by assumption for all integers.

LemmA 3. The subgroups $\left(H_{j}\left(\Omega_{i}\right)\right)_{j \geqslant 1}$ form a Lazard $N$-series in the group $H\left(\Omega_{i}\right)$. Every $H_{j}\left(\Omega_{i}\right)$ is an $\Omega_{j}$-powered group.

\section{Completions of nilpotent groups which may have torsion}

CONSTRUCTION. Let $G$ be a finitely generated nilpotent group of class $c$ with a Lazard $N$-series $\left(G_{j}\right)_{j \geqslant 1}$. For every $j$ choose an ordered basis for $G_{j}$ modulo $G_{j+1}$. Take every element of the basis of $G_{j}$ modulo $G_{j+1}$ to be less than every element of the basis for $G_{j+1}$ modulo $G_{j+2}$. We will call the union of the ordered bases a canonical basis $v_{1}, v_{2}, \ldots, v_{n}$ of $G$. Hence every element of $G$ has a unique representation of the form

$$
v_{1}^{\alpha_{1}} v_{2}^{\alpha_{2}} \ldots v_{n}^{\alpha_{n}}
$$

where for all $i$ we have that $0 \leqslant \alpha_{i}<\varepsilon_{i}$ and $\varepsilon_{i}$ denotes the order of $v_{i}$ modulo $G_{\mu(i)+1}$. Here $v_{i}$ is a member of the chosen basis of $G_{\mu(i)}$ modulo $G_{\mu(i)+1}$.

LEMMA 4. Let $G$ be a finitely generated nilpotent group of class $c$ and $v_{1}, v_{2}, \ldots, v_{n}$ be a canonical basis of $G$. For each $v_{i}$ of the canonical basis we choose an original commutator of weight $\mu(i)$ in a free nilpotent group $F$ of class $c$ and sufficiently large finite rank. Let $H$ denote the subgroup of $F$ generated by all these chosen original commutators. Then the above given association extends naturally to a homomorphism $\varphi$ of $H$ onto $G$. Using the notation of Lemma 2, one also has that

$$
\varphi\left(H_{j}\right)=G_{j} \text { for all } j .
$$


Proof. Let $c_{1}, c_{2}, \ldots, c_{n}$ denote the chosen set of original commutators in $F$, where $\varphi\left(c_{i}\right)=v_{i}$ for all $i$. We define a mapping $\varphi$ of $H$ onto $G$ by

$$
\varphi\left(w\left(c_{l}\right)\right)=w\left(v_{l}\right)
$$

where $w\left(c_{l}\right)$ denotes a word in the generators $c_{1}, c_{2}, \ldots, c_{n}$. Firstly we need to show that $\varphi$ is single-valued. Suppose that $w\left(c_{l}\right)=e$. Then using the commutator collecting process on $w\left(c_{l}\right)$ one gets that

$$
e=w\left(c_{l}\right)=\prod_{v} b_{v}\left(c_{l}\right)^{m_{v}}
$$

where $\left\{b_{v}\left(c_{l}\right), v \in N\right\}$ is the set of weighted basic commutators on the original commutators $c_{1}, c_{2}, \ldots, c_{n}$. By Lemma 2, this representation is unique and so every $m_{v}=0$. Now apply the same commutator collecting process to $w\left(v_{l}\right)$. Then one gets

$$
w\left(v_{l}\right)=\prod_{v} b_{v}\left(v_{l}\right)^{m_{v}}
$$

which is equal to the unit element since every $m_{v}=0$. So $\varphi$ is single-valued. The fact that $\varphi$ is a homomorphism is now an immediate consequence. For every $j$ we have that all the chosen original commutators of weight $j$ belong to $H_{j}$ and hence $G_{j} \subseteq \varphi\left(H_{j}\right)$. Let $b_{v}\left(c_{l}\right)$ be a weighted basic commutator of absolute weight $j$. Then, since $\left(G_{j}\right)_{j \geqslant 1}$ is a Lazard $N$-series, one has that $\varphi\left(b_{v}\left(c_{l}\right)\right)$ belongs to $G_{j}$. Hence $G_{j} \supseteq \varphi\left(H_{j}\right)$.

Notation. We will denote the kernel of the homomorphism $\varphi$ by $K$. So $G \cong H / K$.

Assumption 0 . Suppose that the group $H$ has a canonical basis $u_{1}, u_{2}, \ldots, u_{m}$ relative to the Lazard $N$-series $\left(H_{j}\right)_{j \geqslant 1}$ so that

$$
u_{1}^{\varepsilon_{1}}, u_{2}^{\varepsilon_{2}}, \ldots, u_{m}^{\varepsilon_{m}}
$$

forms a canonical basis for $K$ relative to the Lazard $N$-series $\left(K \cap H_{j}\right)_{j} \geqslant 1$. The numbers $\varepsilon_{i}$ are given in Construction. We use the convention that if the number $\varepsilon_{i}$ is infinite, then $u_{i}^{\varepsilon_{t}}$ is deleted from the above canonical basis.

The above assumption is equivalent to the following

Assumption $0^{\prime}$. There exists a canonical basis $v_{1}, v_{2}, \ldots, v_{n}$ of $G$ so that, for every $i$, if $\varepsilon_{i}$ is finite then $v_{i}^{\varepsilon_{i}}=e$.

Suppose that a finitely generated nilpotent group $G$ has a canonical basis which does not satisfy Assumption $0^{\prime}$. Then it is easy to give some sort of maximal homomorphic image of $G$ which does have a canonical basis satisfying Assumption $0^{\prime}$. 
ExAmpLE. Let $G$ be a finitely generated nilpotent group with Lazard $N$-series $\left(G_{j}\right)_{j \geqslant 1}$. Suppose that $G$ has the following property:

if $g G_{j+1}$ is an element of order $m$ in $G_{j} / G_{j+1}$, then $g$ has order $m$.

Then Assumption 0' holds. One might call such a Lazard $N$-series an unlinked $N$-series. Every Lazard $N$-series of a finite $p$-group of exponent $p$ is unlinked.

DEFINITION 3. Let $R$ be an integral domain which contains $Z$ and suppose that $\varepsilon(>0)$ belongs to $\mathbf{Z}$. Then $\mathbf{Z}$ is said to be $\varepsilon$-isolated in $R$ if and only if $\varepsilon r$ belongs to $\mathbf{Z}$, for some $r$ in $R$, implies that $r$ belongs to $\mathbf{Z}$.

Clearly $\mathbf{Z}$ is $\varepsilon$-isolated in $R$ if and only if no prime divisor of $\varepsilon$ is invertible in $R$.

LEMMA 5. Suppose that Assumption 0 holds and that $\mathbf{Z}$ is $\varepsilon_{i}$-isolated in $\Omega_{\mu(i)}$ for all those $i$ for which $\varepsilon_{i}$ is finite. Then, for the $\left(\Omega_{i}\right)$-completions of $H$ and $K$ given by Theorem 2, one has that

(a) $K\left(\Omega_{i}\right)$ is a normal subgroup of $H\left(\Omega_{i}\right)$;

(b) $K\left(\Omega_{i}\right) \cap H=K$.

Proof. Statement (a) is equivalent to certain polynomial identities, which are true for all integer values of the arguments. This is so since $K$ is normal in $H$. Hence (a) holds. Obviously $K \subseteq H \cap K\left(\Omega_{i}\right)$. Now suppose that

$$
u=\prod_{i} u_{i}^{\varepsilon_{i} a_{i}} \text { belongs to } H,
$$

where for all $i$ we have that $\alpha_{i}$ belongs to $\Omega_{\mu(i)}$. Then $\varepsilon_{i} \alpha_{i}$ belongs to $Z$ and so $\alpha_{i}$ belongs to $\mathbf{Z}$ for all $i$, which gives that $u$ belongs to $K$.

We are now in a position to state the following main result of this paper.

THEOREM 3. Let $G$ be a finitely generated nilpotent group of class $c$. Suppose that

(i) Assumption $0^{\prime}$ holds for some canonical basis $v_{1}, v_{2}, \ldots, v_{n}$ of $G$;

(ii) $\Omega_{1} \subseteq \Omega_{2} \subseteq \ldots \subseteq \Omega_{c}$ is a chain of integral domains such that $Q_{i} \subseteq \Omega_{i}$ for all $i$;

(iii) $\mathbf{Z}$ is $\varepsilon_{i}$-isolated in $\Omega_{\mu(i)}$ for all those i for which $\varepsilon_{i}$ is finite.

Then $G$ is naturally embedded as a subgroup of

$$
H\left(\Omega_{i}\right) / K\left(\Omega_{i}\right)=G\left(\Omega_{i}\right) .
$$

Since $H_{j} \cdot K / K \cong G_{j}$, we denote

$$
H_{j}\left(\Omega_{i}\right) . K\left(\Omega_{i}\right) / K\left(\Omega_{i}\right) \text { by } G_{j}\left(\Omega_{i}\right) .
$$

The series of subgroups $\left(G_{j}\left(\Omega_{i}\right)\right)_{j \geqslant 1}$ is a Lazard $N$-series of $G\left(\Omega_{i}\right)$. Every $G_{j}\left(\Omega_{i}\right)$ is an $\Omega_{j}$-powered group. Every element of $G_{j}\left(\Omega_{i}\right)$ has a unique representation of the 
form $\prod_{i} v_{i}^{\alpha_{i}}$, where $\alpha_{i}$ belongs to a fixed set of coset representatives of $\varepsilon_{i} \Omega_{\mu(i)}$ in $\Omega_{\mu(i)}$ and $\alpha_{i}=0$ if $\mu(i)<j$. Here we take $\varepsilon_{i} \Omega_{\mu(i)}=0$ if $\varepsilon_{i}$ is infinite. Also

$$
G_{j}\left(\Omega_{i}\right) \cap G=G_{j}
$$

and

$$
G_{j}\left(\Omega_{i}\right) / G_{j+1}\left(\Omega_{i}\right) \cong \Omega_{j} \otimes\left(G_{j} / G_{j+1}\right),
$$

for all $j$. Finally, $G\left(\Omega_{i}\right)$ is a Lie algebra over $\Omega_{1}$, where one uses the CampbellHausdorff formula in the way given in Lazard (1954), Chapter II, $\S 4$.

EXAMPLE. Let $G$ be a finite $p$-group of exponent $p$ and class $c<p$. Suppose that

$$
\mathbf{\Omega}_{1} \subseteq \mathbf{\Omega}_{2} \subseteq \ldots \subseteq \mathbf{\Omega}_{c}
$$

is a chain of integral domains so that for all $i$ we have that

$$
Q_{i} \subseteq \Omega_{i}
$$

and $p$ is not invertible in every $\Omega_{i}$. Then for every Lazard $N$-series $\left(G_{j}\right)_{j \geqslant 1}$ of $G$, there exists the completion $G\left(\Omega_{i}\right)$ of $G$ with the above given properties.

It is possible to give a more general version of Theorem 3 for torsion-free finitely generated nilpotent groups. For example, it is not necessary to take a Lazard $N$-series in the group-a central series will suffice. It is also possible to obtain more general versions of Theorems 2 and 3, by taking an ascending chain of integral domains

$$
\mathbf{\Omega}_{1} \subseteq \mathbf{\Omega}_{2} \subseteq \ldots \subseteq \Omega_{n}
$$

such that

$$
Q_{\mu(i)} \subseteq \Omega_{i} \text { for all } i
$$

with

$$
G\left(\Omega_{i}\right)=\left\{\prod_{i=1}^{n} u_{i}^{\alpha_{i}}, \alpha_{i} \in \Omega_{i} \text { for all } i\right\}
$$

in the torsion-free case and

$$
G\left(\Omega_{i}\right)=\left\{\prod_{i=1}^{n} v_{i}^{\alpha_{i}}, \text { every } \alpha_{i} \text { is a representative of } \Omega_{i} \bmod \varepsilon_{i} \Omega_{i}\right\}
$$

for Theorem 3. 


\section{References}

A. M. Duguid (1957), Some generalizations of nilpotent groups (Ph.D. Thesis, University of Cambridge).

M. Hall (1959), The theory of groups (Macmillan, New York).

P. Hall (1957), Nilpotent groups (Canadian Mathematical Congress Notes).

M. Lazard (1954), 'Sur les groupes nilpotents et les anneaux de Lie', Ann. Sci. Ecole Norm. Sup. (3) 71, 101-190.

S. Moran (1973), 'Some subalgebra theorems', J. Algebra 27, 366-371.

R. B. Warfield, Jr. (1976), Nilpotent groups (Lecture Notes in Mathematics 513, Springer-Verlag, Berlin).

The University

Canterbury

Kent

United Kingdom 